\title{
Magnetostatic bias in a composite hard/soft/hard microlayer
}

\author{
L. Kraus \\ Institute of Physics, ASCR, 18221 Prague, Czech Republic \\ K. R. Pirota \\ Applied Physics Department, Autonomous University of Madrid, 28049 Madrid, Spain \\ J. Torrejón and M. Vázquez ${ }^{a)}$ \\ Institute of Materials Science, CSIC, 28049 Madrid, Spain
}

(Received 3 August 2006; accepted 21 January 2007; published online 26 March 2007)

A composite magnetically hard/soft/hard trilayer ribbon with micrometric thickness has been prepared by combined melt-spinning and electroplating techniques. Its main peculiarities relate to the existence of a magnetic coupling between hard and soft magnetic phases resulting in bias magnetic field acting on the soft layer. A systematic study of the low-field hysteresis loops has been performed after premagnetizing under a direct current saturating field as a function of the geometrical dimensions of both types of layers. The analysis of the observed bias effect has allowed us to conclude the magnetostatic origin of the tailorable coupling. The present investigation in microscale system points out to the potential technological applications that in a further step can be developed in nanomagnetic systems. (C) 2007 American Institute of Physics.

[DOI: $10.1063 / 1.2713363$ ]

\section{INTRODUCTION}

Magnetic bias is a powerful tool for obtaining unique properties of multicomponent magnetic systems currently used in various applications. For example, multilayered planar structures can exhibit attractive phenomena related to the exchange coupling at ferro/antiferromagnetic interfaces, or "exchange bias," which are used in spin-valve devices for reading heads, in magnetic sensors and biosensors, or in magnetic random access memories. ${ }^{1}$ Exchange bias in partially surface crystallized amorphous wires or ribbons can be used in security $\operatorname{tags}^{2}$ or giant magnetoimpedance valves. ${ }^{3}$

In multilayered or nanogranular systems three main types of magnetic interactions can be recognized: (a) exchange coupling between touching (ferro/antiferro or ferro/ ferromagnetic) phases, (b) indirect Ruderman-Kittel-KasuyaYosida (RKKY)-like coupling through an interfacial nonmagnetic metallic layer, and (c) long-range dipolar coupling. The main feature of magnetic bias is the unidirectional character of anisotropy that results in a shift of hysteresis loop away from the zero-field axis. ${ }^{4}$ The orientation of this shift, labeled as bias field, $H_{b}$, can be either parallel or antiparallel to the "frozen-in" spin direction of the pinning phase (antiferromagnetic or hard ferromagnetic-like, respectively) depending on whether the bias coupling is ferro- or antiferromagnetic in nature.

Dipolar magnetic bias has been recently suggested to interpret the giant magnetoimpedance behavior of certain amorphous microwires. ${ }^{5}$ A currently employed processing method to achieve biasing effect, field annealing in air used for $\mathrm{CoFeSiB}$ ribbons, ${ }^{2,3}$ is nevertheless applicable only to a narrow range of chemical compositions and is not fully reproducible. In addition, intragranular magnetostatic coupling

${ }^{a)}$ Electronic mail: mvazquez@icmm.csic.es has been considered to understand the enhanced hard magnetic behavior of soft/hard composite permanent magnets. ${ }^{6}$

Combined melt-spinning and electrodeposition techniques have been used for the preparation of multilayered systems with controlled parameters. ${ }^{7}$ A particular type of trilayered system has been recently proposed as a sensing element for temperature sensing making use of the different expansion coefficients of nucleus and outer layers. ${ }^{8}$

The objective of this work has been the study of the magnetic bias phenomenon in a magnetically hard/soft/hard micrometric multilayer composed of an ultrasoft magnetic Co-rich amorphous ribbon covered on both sides by magnetically harder layers of electroplated CoNi alloy. A particular motivation of the work has been to find an alternative way of biasing soft magnetic amorphous ribbons which can be of further interest in nanosystems.

\section{SAMPLES PREPARATION}

A precursor amorphous ribbon with nominal composition $\mathrm{Co}_{67} \mathrm{Fe}_{4} \mathrm{Cr}_{7} \mathrm{Si}_{8} \mathrm{~B}_{14}, 1.0 \mathrm{~mm}$ wide and $20 \mu \mathrm{m}$ thick, was prepared by planar flow casting. This alloy composition was selected because of its vanishing magnetostriction constant, of the order of $1 \times 10^{-7}$, resulting in an ultrasoft magnetic character where the influence of magnetoelastic interactions is reduced. Pieces of amorphous samples with length, $L_{s}$, between 3.5 and $7.0 \mathrm{~cm}$ were cut from the as-quenched ribbon.

To obtain the aimed trilayer structure, two magnetically harder CoNi layers have been electroplated on both sides of the melt-spun soft amorphous ribbon. The electroplating was done using an aqueous solution of $\mathrm{NiSO}_{4} \cdot 6 \mathrm{H}_{2} \mathrm{O}(150 \mathrm{~g} / \mathrm{l})$, $\mathrm{CoSO}_{4} \cdot 7 \mathrm{H}_{2} \mathrm{O}(150 \mathrm{~g} / \mathrm{l}), \mathrm{H}_{3} \mathrm{BO}_{3}(45 \mathrm{~g} / \mathrm{l}), \mathrm{CoCl}_{2} \cdot 7 \mathrm{H}_{2} \mathrm{O}(22.5$ $\mathrm{g} / \mathrm{l})$, and $\mathrm{NiCl}_{2} \cdot 7 \mathrm{H}_{2} \mathrm{O}(22.5 \mathrm{~g} / \mathrm{l})$, and it was carried out in a constant current density regime at $40{ }^{\circ} \mathrm{C}$, using a special cell which details can be found elsewhere. ${ }^{7}$ The ribbon was 


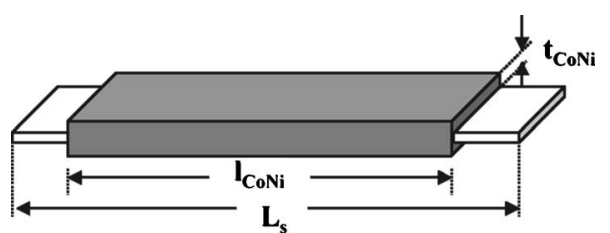

FIG. 1. Schematic view of the multilayer: the soft CoFeCrSiB amorphous nucleus (white) is covered by the hard CoNi layer (gray).

placed in the center of the cell, previously positioned in the vertical orientation. A current density of $12 \mathrm{~mA} / \mathrm{cm}^{2}$ was used in the present experiments for which the composition of the plated alloy was $\mathrm{Co}_{83} \mathrm{Ni}_{17}$, as deduced by glow discharge optical emission spectroscopy technique. ${ }^{9}$ X-ray diffraction analysis unveils its hexagonal-closed-packed crystalline structure. The composition is nearly nondependent on the deposition time, which was varied from 1 to $60 \mathrm{~min}$. A nearly linear increase of the thickness of the electroplated layer with deposition time is obtained as determined by optical microscopy. The thickness, $t_{\mathrm{CoNi}}$, of the investigated hard layers ranged between 0.4 and $24 \mu \mathrm{m}$, with a growth rate of around $0.4 \mu \mathrm{m} / \mathrm{min}$. The length of electroplated CoNi layers, $l_{\mathrm{CoNi}}$, ranged between 1.5 and $5.5 \mathrm{~cm}$, and the lateral thickness of hard layer is reduced to about $1 \mu \mathrm{m}$. A schematic view of the multilayer is shown in Fig. 1 where the central layer (in white) represents the amorphous nucleus and the external layers (in gray) denote the external CoNi outer layers.

\section{MAGNETIC MEASUREMENTS}

The hysteresis loops of the trilayer system were measured by a personal computer controlled quasistatic loop tracer with a pick-up coil $8 \mathrm{~mm}$ long. The high-field hysteresis loops are plotted in Fig. 2(a) for selected samples with different thickness of the hard layers. Each hysteresis loop can be decomposed into two parts: (i) A narrow low-field magnetization process, which can be ascribed to the magnetization reversal of the soft magnetic phase of the precursor amorphous ribbon and (ii) a wider one which is due to the reversal at the hard magnetic electroplated CoNi layers. While the amorphous component is practically saturated at low magnetic field (less than $100 \mathrm{~A} / \mathrm{m}$ ), the full saturation of the CoNi layer cannot be reached even at the highest field available in our equipment $(60 \mathrm{kA} / \mathrm{m})$. a)

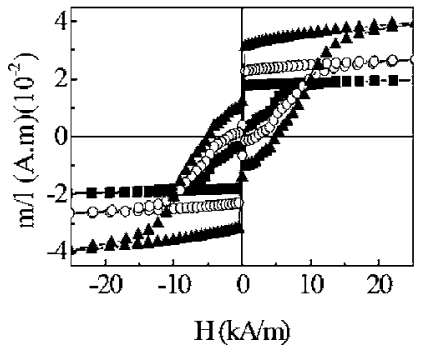

b)

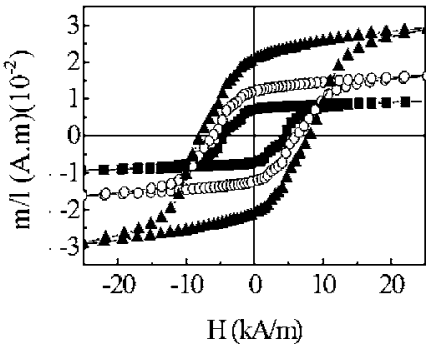

FIG. 2. High-field hysteresis loops of soft/hard bi-phase microlayer for different thickness of the electroplated hard CoNi layer: $6 \mu \mathrm{m}(\boldsymbol{\square}), 12 \mu \mathrm{m}$ (○), and $24 \mu \mathrm{m}(\boldsymbol{\Delta})(\mathrm{a})$; and loops corresponding to the CoNi hard layers (b) $\left(L_{s}=7 \mathrm{~cm}, l_{\mathrm{CoNi}}=5.5 \mathrm{~cm}\right)$.

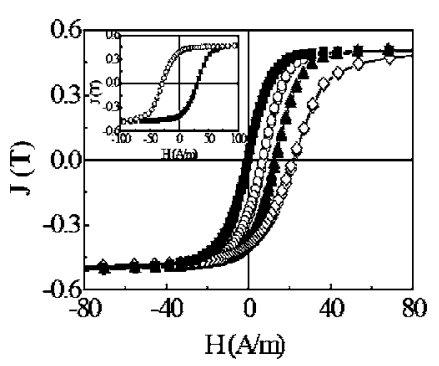

FIG. 3. Low-field hysteresis loops of the soft $\mathrm{CoFeCrSiB}$ inner layer after premagnetizing in a saturating field of $+40 \mathrm{kA} / \mathrm{m}$ for a range of different thicknesses of the outer CoNi hard layer: $0.4 \mu \mathrm{m}(\boldsymbol{\square}), 6 \mu \mathrm{m}(\bigcirc), 12 \mu \mathrm{m}(\mathbf{\Delta})$, and $24 \mu \mathrm{m}(\diamond)\left(L_{s}=7 \mathrm{~cm}, l_{\mathrm{CoNi}}=5.5 \mathrm{~cm}\right)$. The inset shows the loops after saturation in $+40 \mathrm{kA} / \mathrm{m}(\square)$ and $-40 \mathrm{kA} / \mathrm{m}(\bigcirc)$ for a hard layer $24 \mu \mathrm{m}$ thick.

In order to deeper analyze the magnetic characteristics of the CoNi electroplated hard phase, an appropriate step function (at $H=0$ ) has been subtracted from the loops shown in Fig. 2(a) resulting in the hysteresis loops observed in Fig. 2(b). The magnetic moment per unit length, $\mathrm{m} / \mathrm{l}$, measured at $H=60 \mathrm{kA} / \mathrm{m}$ and close to the saturation value (not shown here), increases quasilinearly with the deposition thickness which confirms the linear increase of the CoNi thickness with time of electroplating. ${ }^{9}$ Concerning the magnetic hardness of the electroplated layers, its coercivity increases monotonously with the deposition time up to approach a saturation value of around $10 \mathrm{kA} / \mathrm{m}$.

The low-field hysteresis loops are plotted in Fig. 3 after saturating in a strong positive magnetic field $(+40 \mathrm{kA} / \mathrm{m})$. These hysteresis loops can be taken as corresponding exclusively to the soft magnetic amorphous phase since in this low-field region the magnetization of hard magnetic CoNi layers is practically constant around its remanence state.

The remarkable intrinsic ultrasoft magnetic character of the amorphous ribbon should be first mentioned, with intrinsic coercivity takes values of the order of $1 \mathrm{~A} / \mathrm{m}$. Nevertheless, the most noticeable feature is the observed shift of the loops along the horizontal axis which increases with the thickness of the hard layers, and actually reflects the existence of a bias field, $H_{b}$. In addition, the orientation of that bias field depends on the magnetic state of the hard magnetic layers as is confirmed when a strong negative saturation field $(-40 \mathrm{kA} / \mathrm{m})$ is applied before the measurement: as observed in the inset of Fig. 3, the asymmetry of the hysteresis loop is now reversed.

In order to obtain detailed information on the magnetic interaction between soft and hard layers further experiments have been performed with different geometrical dimensions of the layers. Figure 4(a) shows the loops of the soft layer with different length, $L_{s}$, ranging between 3.5 and $7.0 \mathrm{~cm}$, after premagnetizing at a saturating positive field $(+40 \mathrm{kA} / \mathrm{m})$. The length, $l_{\mathrm{CoNi}}$, and thickness, $t_{\mathrm{CoNi}}$, of hard layers are kept constant at $1.75 \mathrm{~cm}$ and $12 \mu \mathrm{m}$, respectively. As can be observed, the hysteresis loops are characterized by two-step regions with different susceptibility. The susceptibility in the lower-field region, $\chi_{1}$, decreases when the length of the soft layer is reduced, which seemingly is a conse- 

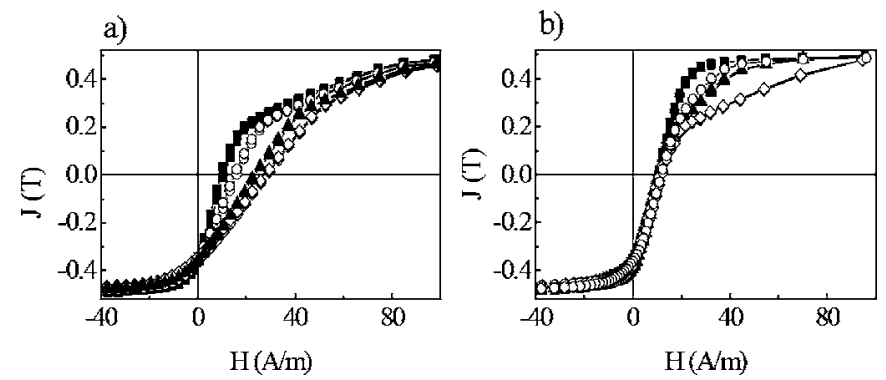

FIG. 4. Low-field hysteresis loops of the soft $\mathrm{CoFeCrSiB}$ inner layer after premagnetizing in a saturating field of $+40 \mathrm{kA} / \mathrm{m}$ for a range of different length of the soft layer, $L_{s}: 7 \mathrm{~cm}(\boldsymbol{\square}), 5.12 \mathrm{~cm}(\bigcirc), 3.93 \mathrm{~cm}(\boldsymbol{\Delta})$, and 3.52 $\mathrm{cm}(\diamond)\left(l_{\mathrm{CoNi}}=1.75 \mathrm{~cm}, t_{\mathrm{CoNi}}=12 \mu \mathrm{m}\right)(\mathrm{a})$, and of the hard layer, $l_{\mathrm{CoNi}}: 4.5$ $\mathrm{cm}(\square), 3.4 \mathrm{~cm}(\bigcirc), 2.65 \mathrm{~cm}(\boldsymbol{\Delta})$, and $1.75 \mathrm{~cm}(\diamond)\left(L_{s}=7 \mathrm{~cm}, t_{\mathrm{CoNi}}\right.$ $=12 \mu \mathrm{m})(\mathrm{b})$.

quence of the corresponding increase of its demagnetizing field. In turn, the susceptibility in the higher-field region, $\chi_{2}$, remains practically constant.

Figure 4(b) shows the loops as a function of the length of the hard layer, $l_{\mathrm{CoNi}}$, for constant values of the soft layer length, $L_{s}=7 \mathrm{~cm}$, and the thickness of the hard layers, $t_{\mathrm{CoNi}}$ $=12 \mu \mathrm{m}$. Now, the lower-field susceptibility, $\chi_{1}$, is nearly constant while the higher-field susceptibility, $\chi_{2}$, decreases with the length of the hard layer. In fact, the two susceptibility regions are only detected when the length of hard layer is short enough.

\section{ABOUT THE ORIGIN OF THE MAGNETIC COUPLING}

The hysteresis loops are shifted along the same direction as that of the previously applied saturating field which indicates the existence of a coupling with similar characteristic to that of an antiferromagnetic-like coupling. This type of interaction can be expected in principle for a short range ferro/antiferromagnetic exchange coupling at the hard/soft magnetic interface as well as for a long-range magnetostatic coupling of the soft magnetic phase with the stray fields of magnetic charges on the hard magnetic layers.

It is well known that the exchange bias in ferro/ antiferromagnetic (AFM) bilayers only extends a limited distance and that it does not depend on the AFM layer for thickness above some critical value (few nanometers). ${ }^{10}$ Consequently, the origin of the observed bias field can be easily confirmed from an analysis of the dependence of $H_{b}$ on the thickness of the hard magnetic layer. Figure 5 summarizes the dependence of the bias field on the thickness of hard layers and on the length of the soft layer. As observed, the bias field is nearly proportional to the thickness of the hard magnetic layer which indicates that magnetostatic coupling is actually responsible for the magnetic bias. Moreover, the bias field decreases with the length of the soft layer that suggests that its intrinsic demagnetizing field also plays a noticeable role.

The nearly saturating premagnetizing field, $H_{\text {sat }}$, creates net magnetic charges at the ends of the external CoNi layers. These uncompensated charges remain active during the whole low-field magnetization/remagnetization process of

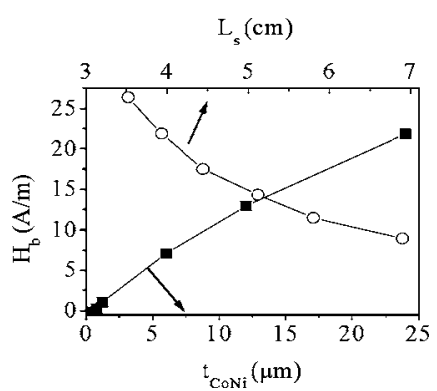

FIG. 5. The bias field, $H_{b}$, of the soft phase as a function of the thickness of the hard magnetic layers, $t_{\mathrm{CoNi}},\left(L_{s}=7 \mathrm{~cm}, l_{\mathrm{CoNi}}=5.5 \mathrm{~cm}\right)(\boldsymbol{\square})$, and of the length of the soft layer, $L_{s},\left(t_{\mathrm{CoNi}}=12 \mu \mathrm{m}, l_{\mathrm{CoNi}}=1.75 \mathrm{~cm}\right)(\bigcirc)$.

the ultrasoft central layer, when the applied field does not modify significantly the domain structure of the hard phase.

Such uncompensated charges originate a magnetostatic field in the soft amorphous layer which is antiparallel to magnetization in the hard layers. We propose that the observed bias field, $H_{b}$, corresponds to that magnetostatic field. Moreover, its strength, related to the net magnetic charges at the ends of the hard layers, is confirmed to be proportional to their thickness as derived in Fig. 5.

Based on the results shown in Fig. 4, the remagnetization process of the ultrasoft layer can be schematically followed in Fig. 6. The arrows denote the magnetization direction in the soft (white) and hard (gray) layers. Zones 1 and 4 of the hysteresis loop correspond to the magnetically saturated states of the soft phase, while in zones 2 and 3 the effective magnetization partially rotates from the axial orientation. The biphase system is first submitted to a positive saturating applied field. Then, the applied field is moved to a slightly negative value (zone 1). There, the ultrasoft layer saturates at very low-field in the negative orientation under the additional action of the magnetostatic field of the hard layer.

Two spatial regions are proposed within the soft layer determined by the strength of that magnetostatic field responsible for the different susceptibility in zones 2 and 3. The external region exhibits larger susceptibility due to the reduced effect from the stray field of the hard layers, and is mainly responsible for susceptibility, $\chi_{1}$, in zone 2 of the

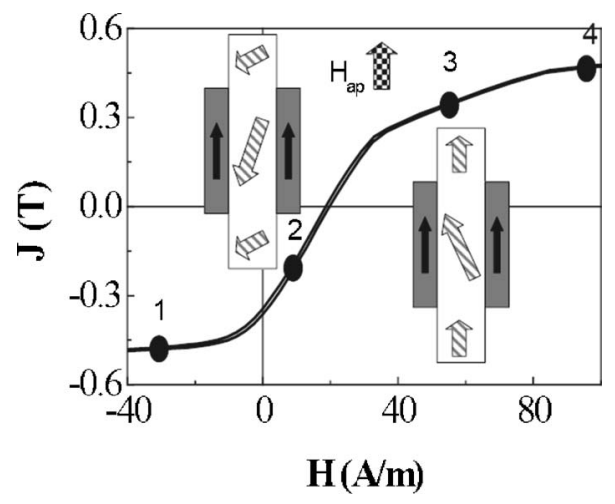

FIG. 6. Schematic view of the remagnetization process of the soft layer through four stages, under the magnetostatic interaction between the two magnetic phases. The arrows denote the magnetization of the soft (white) and hard (gray) layers corresponding to nonsaturated stages 2 and 3 . 
loop. When the length of the soft layer is reduced, its demagnetizing factor increases and $\chi_{1}$ decreases. For higher applied field (zone 3 of the loop), the external region is nearly saturated and the central region of the soft layer mainly contributes to the observed susceptibility, $\chi_{2}$. The two regions in the soft layer with different susceptibility are only detected when its length is significantly longer than that of the hard layer. The existence of such two regions is a consequence of the inhomogeneity of the magnetostatic field induced by the hard layers.

\section{CONCLUSIONS}

A trilayer system with hard/soft/hard magnetic behavior has been prepared by combined melt-spinning and electrodeposition techniques. After premagnetizing to saturation, the low-field hysteresis loop of the ultrasoft layer is magnetically observed to be biased. From the study of the influence of the dimensions of soft and hard layers, the antiferromagnetic-like bias field has been confirmed to be magnetostatic in nature. Its origin is ascribed to unbalanced magnetic charges at the ends of the hard layer.

The reported technique to fabricate these multilayer systems can be used to tailor the magnetic permeability of a material by selective electroplating of the hard layer. The low-field magnetization curve presents a practically nonhysteretic character which suggests an additional interest of these materials as sensing elements in sensor field devices.
Finally, nanoscaled miniaturization of these multilayer systems can open additional technological applications.

\section{ACKNOWLEDGMENTS}

The authors are grateful to Dr. Dušan Janičkovič of the Institute of Physics, Slovak Academy of Sciences, Bratislava for supplying the amorphous ribbons and to Dr. Giovanni Badini-Confalonieri of the Instituto de Ciencias de Materiales, Madrid for helpful discussions. The work has been supported by the Czech research Project No. AVOZ10100520 (L.K.) and Spanish research Project No. MAT2004-00150.

${ }^{1}$ J. Nogués, J. Sort, V. Langlais, V. Skumryev, S. Suriñach, J. S. Muñoz, and M. D. Baró, Phys. Rep. 422, 65 (2005).

${ }^{2}$ K. H. Shin, C. D. Graham, Jr., and P. Y. Zhou, J. Magn. Magn. Mater. 177-181, 225 (1998)

${ }^{3}$ C. G. Kim, K. J. Jang, H. C. Kim, and S. S. Yoon, J. Appl. Phys. 85, 5447 (1999).

${ }^{4}$ W. H. Meiklejohn and C. P. Bean, Phys. Rev. 105, 904 (1957).

${ }^{5}$ J. P. Sinnecker, A. de Araujo, R. Piccin, M. Knobel, and M. Vázquez, J. Magn. Magn. Mater. 295, 121 (2005).

${ }^{6}$ A. M. Gabay, M. Marinescu, and G. C. Hadjipanayis, J. Appl. Phys. 99, 08B506 (2006)

${ }^{7}$ K. Pirota, M. Hernández-Vélez, D. Navas, A. Zhukov, and M. Vázquez, Adv. Funct. Mater. 14, 266 (2004).

${ }^{8}$ P. Mendoza-Zelis, F. Sánchez, and M. Vázquez, J. Appl. Phys. 101, 034507 (2007).

${ }^{9}$ K. Pirota, M. Provencio, K. García, P. Mendoza, M. Hernandez-Velez, and M. Vázquez, J. Magn. Magn. Mater. 290-291, 68 (2005).

${ }^{10}$ J. Nogués and I. K. Schuller, J. Magn. Magn. Mater. 192, 203 (1999). 